\title{
Low-Latency Science Exploration of Planetary Bodies: A Demonstration using ISS in Support of Mars Human Exploration
}

\author{
Harley Thronson ${ }^{1}$, Azita Valinia ${ }^{2}$, Jacob Bleacher ${ }^{3}$, Jennifer Eigenbrode ${ }^{4}$, Jim Garvin ${ }^{5}$, Noah Petro ${ }^{6}$ \\ Science and Exploration Division, NASA Goddard Space Flight Center, Greenbelt, MD 20771
}

We summarize a proposed experiment to use the International Space Station to formally examine the application and validation of low-latency telepresence for surface exploration from space as an alternative, precursor, or potentially as an adjunct to astronaut "boots on the ground." The approach is to develop and propose controlled experiments, which build upon previous field studies and which will assess the effects of different latencies (0 to $500 \mathrm{msec}$ ), task complexity, and alternate forms of feedback to the operator. These experiments serve as an example of a pathfinder for NASA's roadmap of missions to Mars with low-latency telerobotic exploration as a precursor to astronaut's landing on the surface to conduct geological tasks.

\section{Introduction and Background}

$\mathrm{R}$ obotic systems are rapidly becoming increasingly sophisticated, versatile, and widely accepted as key elements of ambitious space exploration [REF needed]. They are able to carry out complex investigations in locations that may forever be too dangerous or expensive for human explorers. Robots are far less costly than astronauts and are seen by many - perhaps especially among younger people - as full participants in the process of exploration of the cosmos. The one characteristic that robots lack, however, is anything approaching human intelligence, creativity, and inventiveness. That limitation on Earth is readily overcome by having human operators in close proximity to the robot, only a fraction of a second away in light-travel time; that is, low-latency operation.

Low-latency telerobotics (LLT) is being widely assessed and advocated as a means of planetary exploration via "human telepresence" as an alternative or precursor to the much more expensive landing and surface operation by humans. In these telepresence scenarios, human explorers extend human cognition to the surface of Moon, Mars, and other planetary bodies without the risk and expense of exposing humans to hazardous conditions on surfaces, as well as the dangers of landing and returning to orbit from within deep gravity wells. Human telepresence may also include humans in a safe location on the surface with the rovers deployed to more remote or hazardous locations. In all cases, "telepresence exploration" humans remain in locations where control of robots on planetary surfaces is limited by round-trip light travel times to less than about $500 \mathrm{~ms}$. That is, low latency, as opposed to the highlatency surface investigations involving tens of minutes or hours such as in the operation of NASA's Mars Science Laboratory Curiosity rover. Given the significant expense of safely landing and operating humans on a planetary surface, such as on the inhospitable martian surface, it may well be, as we have argued elsewhere ${ }^{1}$, that telepresence exploration could enable humans to travel to Mars long before it is possible to land them. To have this option will require hardware development and operations tests designed for low-latency communications in order to assess the potential role of human telepresence in mission activities and how it can best be utilized.

The International Space Station (ISS) provides an excellent platform to test the validity and ultimately the scientific usefulness of LLT exploration investigations from orbit as there are no other facilities that so closely mimic future LLT operations scenarios (e.g., provide long-duration zero gravity, significant limits to astronaut time and attention, constrained physical environment, high-bandwidth communication whose latency can be manipulated). The first engineering-oriented LLT experiment was carried out in summer, 2013, by a team led by NASA Ames Research Center ${ }^{2}$, which demonstrated the basic task of assessing the topology of the landscape and deploying an antenna on the ground by robots operated by astronauts from ISS.

\footnotetext{
${ }^{1}$ Senior Scientist for Advanced Concepts in Astrophysics, NASA Goddard Space Flight Center, AIAA Member

${ }^{2}$ Associate Director for Research \& Development, Sciences and Exploration Directorate, NASA Goddard Space Flight Center, AIAA member

${ }^{3}$ Staff Planetary Geologist, NASA Goddard Space Flight Center

${ }^{4}$ Staff Space Scientist, NASA Goddard Space Flight Center,

${ }^{5}$ Chief Scientist, NASA Goddard Space Flight Center

${ }^{6}$ Staff Geoscientist, NASA Goddard Space Flight Center, AIAA member?
} 
LLT activities and processes are not well established within the current domains of terrestrial field sciences (i.e., geology and astrobiology) nor do the proper low latency or "real-time" science instruments exist to take advantage of this capability. Therefore, any high-fidelity experiments involving ISS crew and JSC mission control operations require controlled testing and evaluation on the ground to baseline performance in advance of space-based implementation (and evaluation of performance tied to science value or impact).

In this paper, we describe a follow-on concept of operation in which the astronauts on orbit carry out a robotic geological field experiment as a demonstration of an alternative or adjunct to in situ exploration directly on the planetary surface by astronauts. The experiment will be compared to control experiments in high-latency telerobotics (HLT) such as by means of Curiosity on Mars, as well as prior analog field campaigns such as Desert Research And Technology Studies, the Arctic Mars Analog Svalbard Expedition (AMASE), Pavilion Lake, or NASA Extreme Environment Mission Operations (NEEMO) [REFs needed].

\section{Telepresence Exploration: the ISS as Testbed}

We propose that the ISS be used as a test bed to approximate low-latency telerobotic operation in orbit around the Moon and, especially at Mars. It is unclear how LLT would be utilized in future human exploration of the Solar System. A number of scenarios can be assessed, including, but not limited to: 1) exclusively human telepresence on a planet's (or satellite's) surface as operated from orbit (or a moon), 2) telepresence on a planet's surface from elsewhere on the surface to investigate dangerous or special regions of interest, 3) a combination of telepresence and human presence, 4) and any of the previous scenarios with multiple telerobotically operated assets. Furthermore, one might consider combinations of LLT with not only human presence, but also HLT assets as well. Thus, the trade space is rich for consideration of the role that low latency might play in future planetary exploration.

Human presence and HLT exploration missions have been conducted (for instance Apollo and Curiosity) and, as such, high-fidelity data exist for trade studies related to their roles in future human exploration. However, LLT telerobotics has at best been evaluated in low-fidelity NASA tests. Therefore, high-fidelity LLT tests are necessary to provide comparable data for evaluation of a low-latency approach to human exploration.

There exist at present some basic LLT activities that can be tested in a flight environment on ISS in the near term. Such tests should build upon the success of the 2013 test, and be used to establish a baseline for conducting field science that involves LLT. The most fundamental test is to develop a timeline for LLT scientific exploration with currently available hardware and instruments. Although NASA has developed science instruments for decades, instruments specifically developed to enable or take advantage of low-latency operations do not exist. This issue is not limited to instrumentation. High-fidelity LLT tests that establish a timeline for operations with currently existing hardware and software will provide preliminary data for trade studies that seek to identify what technologies should be enhanced and/or newly developed in order to make LLT operations a viable option. Meanwhile, baseline operations tests of LLT will provide insight into how LLT efforts can be used or merged with other strategies to best maximize science productivity in future missions

Initial tests should be designed to assess the effect of LLT on basic science tasks that would likely be repeated frequently during surface operations. Metrics for such an experiment would be used in evaluating the experiment against other techniques: for example, execution time for each step in an experiment versus total execution time for the experiment; efficiency in terms of time and resources to determine the next step in the chain of experiments, the number of people needed to execute each task, as well as whether each task was executed correctly and whether the expected scientific result - known only to the experiment designer - was achieved.

Specific metrics include

1. Time to carry out each individual step in a science work plan

2. Number of cognitive steps needed to carry out the task

3. Total time expended to execute the task

4. The efficiency of how "the next step" was derived

5. The number involved to execute the task (e.g., operator and advisor(s))

6. Correct execution of the task (i.e., success criteria)

If science measurements were taken, did they render expected results? This is a key question since most metrics are designed for evaluating process efficiency rather than getting the "right" answer. Both need to be addressed.

Upon establishment of a basic timeline for LLT surface science operations, additional variables can be assessed to evaluate the characteristics of a successful LLT operation. For example, different latencies may be introduced into the experiment, alternative feedback systems can be employed, and variation in signal bandwidth (e.g., variable data rates) will be adopted. Different levels of precision and delicacy of surface manipulation may be explored and unexpected events can be introduced to the pre-planned telerobot operations. Experiments should be designed to 
duplicate the constrained times that actual orbiting astronauts will one day face: accomplishing challenging tasks before, for example, the spacecraft passes out of communication link with the surface robot. Thus, hybrid LLT/HLT scenarios might be highly desirable where astronauts operate a rover in LLT and prepare it for HLT measurements while the orbiting spacecraft is out of communication with the surface asset. Depending upon the available resources, these experiments may include one or more partner "astronauts" on the ground working with the telerobot. This will permit an evaluation of scenarios for effective close-proximity astronaut/telerobot partnership to carry out plausible science-related tasks. Finally, testing can assess varying amounts of autonomy in the telerobot, that is, how tightly linked are the control of the robot to the human in space,

ISS provides the only high-fidelity opportunity to conduct LLT analog tests that can help provide a comparable characterization of the trade space with human presence and HLT. Development of a basic timeline for LLT surface science operations should serve as the starting point for such an effort, followed by more complex testing of different variables involved in telepresence. Thus, to best establish an approach to future human spaceflight that might include any or all three of these approaches (human presence, HLT, LLT) an ISS campaign to evaluate the role of telepresence in science operations is critical. Due to the success of the 2013 LLT engineering test, now is the time to implement such a plan so that momentum gained from software integration and hardware development to enable that test is not lost or minimized.

\section{Additional Considerations}

As part of our activities, advances in IT and telerobotics should be forecast against future desired scientific capabilities driven by published priorities and specific investigation scenarios [REF to Decadal Surveys]. For example, in the case of surface exploration of Mars it must be ensured that LLT-based approaches considered today (2014-2106) are not "out-dated before their time" in the mid-2020s and 2030s. A study of how robotic automation and utilization for surface scientific investigations in light of predicted capabilities (i.e., Moore's law and others) and economic drivers may be undertaken to forecast deep-space human spaceflight activities in the 2030s in order to identify where LLT is most appropriate [REF to Wilcox et al].

We believe that it is scientifically valuable over the next few years to rigorously quantify cost/benefits of LLT for future planetary surface science priorities enabled by human space flight with or without LLT at a given destination. Experiments that serve to identify a few LLT scenarios relevant to future human exploration science activities in the 2020 s and 2030s that can be evaluated via ISS would be invaluable as validation of the premise that new science can be achieved. However, it is important to identify when the ISS-telerobotic system is required in contrast with "nice to have."

\section{References}

\footnotetext{
${ }^{1}$ Paper by Dan

2 Terry Fong paper? HET?

Papers by Jake, Jen, MSL (Grotz), Dan Lester, Oceaneering, et al.
} 